\title{
On The Rapid Cryoimmobilization Of Bulk Specimens For "Quality" Macromolecular Imaging By Low Temperature High Resolution SEM
}

\author{
R.P. Apkarian
}

Integrated Microscopy \& Microanalytical Facility/ Department of Chemistry Emory University 1515 Dickey Dr. Atlanta, GA 30322

Rapid cryo-immobilization of bulk specimens such as hydrogels, macromolecular complexes and cell suspensions $(2-10 \mu \mathrm{l})$ in which the water content is cooled into the vitreous state should be strived for when low temperature (LT) HRSEM is employed. Just as in cryo-TEM preparations of thin specimens vitrified in $100 \mathrm{~nm}$ thick films, the first few hundred nanometers of the specimen should be non crystalline when imaged by LTHRSEM. Still it is not known whether the water in our samples can be regarded as crystalline ice, vitreous water, or rather an ambiguous amorphous solid. The problem one faces when a minute bulk sample is cooled for LTHRSEM studies is that one can never be absolutely certain that the cryospecimen surface contained vitreous water since electron diffraction cannot be performed on bulk specimens as is done with $100 \mathrm{~nm}$ thick specimens in a cryo-TEM [1]. Nonetheless by employing very rapid cryoimmobilization, using ethane plunge and high pressure freezing (HPF) of very small specimens, with or without high vacuum etching and ultrathin metal coatings, formation of specimen damaging hexagonal ice may be minimized. Careful assessment of the cryospecimen facilitated quality LTHRSEM analysis of cell suspensions and macromolecular complexes [2-4].

Elastin-mimetic triblock copolymer hydrogels $(1,10, \& 25 \mathrm{wt} \%)$ and self-assembled amyloid fibers $(3 \mathrm{mg} / \mathrm{ml})$ were loaded into Balzers gold planchets, plunge cooled in ethane $\left(-183^{\circ} \mathrm{C}\right)$ and by $\mathrm{HPF}$, then loaded onto a Gatan CT-3500 cryostage under $\mathrm{LN}_{2}$. A prechilled knife was used to lightly fracture the cold sample prior to transfer into a Denton DV-602 high vacuum chamber for etching and Cr coating (1-2 nm thickness). The stage was transferred in-lens of a DS-130 FESEM/STEM and imaged with 5-30 keV. Recordings (5 \& 17 Mbytes) were made in tif format.

Genetically engineered elastin triblock copolymers that formed hydrogels at $25 \mathrm{wt} \%$ were extensively studied by LTHRSEM [2-4]. Casting the elastin copolymer from $\mathrm{H}_{2} \mathrm{O}$ at lower concentrations $(1 \& 10 \mathrm{wt} \%)$ exhibited remarkable preservation of the honeycomb motif and macromolecular fibrils (FIG. 1). It was evident that the \% wt of elastin compound determined the hydrogel pore size while high magnification LTHRSEM revealed the same structural $20 \mathrm{~nm}$ triblock nodes and $\sim 7 \mathrm{~nm}$ elastin-like filaments. In unetched elastin specimens, $20 \mathrm{~nm}$ nodes were observed but no ramified crystalline shapes were seen even in the 1\% hydrogel. LTHRSEM of amyloid fibril suspensions revealed a glass-like specimen that contained fibrils (FIG. 2). Particulates and fibrils $20 \mathrm{~nm}$ in diameter characterized the hydrated amyloid proteins.

Cryoprotection agents were not employed in these protein samples. Elastin protein hydrogels were well preserved at all concentrations and no evidence for damaging crystalline ice was observed using either freezing method. Although electron diffraction data on the state of cooled water in these samples was not available, a featureless aqueous phase remained as the filler in the pores of elastin hydrogels. Fibers and nodes 7-20 nm in width was the characteristic molecular architecture of the pore walls and protein concentration was the determinant of pore diameter. Amyloid fibril 
assemblies were observed by cryoetch LTHRSEM using the ethane plunge method and shallow fracture. Ice crystals of any size were never observed by LTHRSEM but presence of vitrified water was not a certainty.
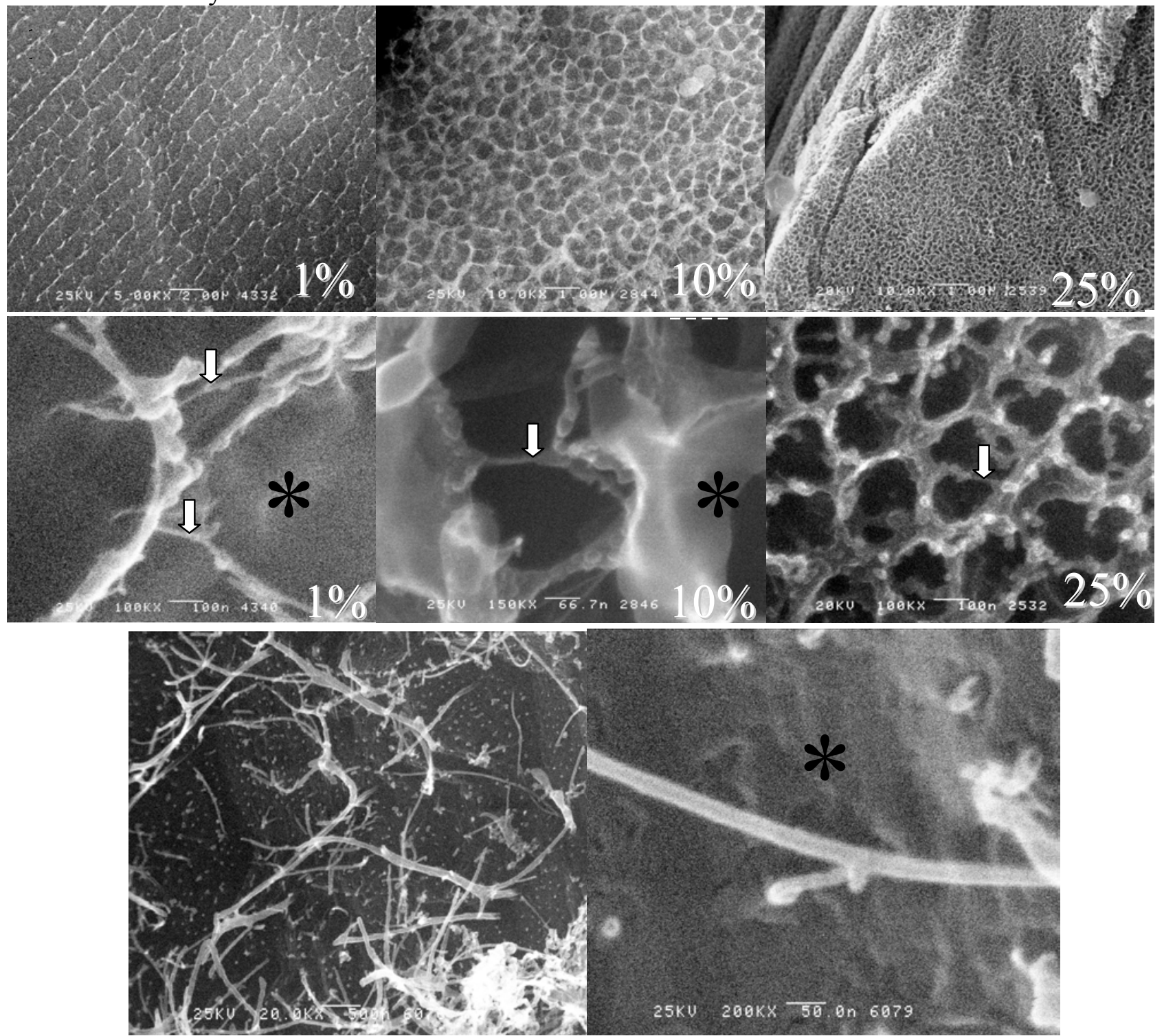

FIG. 1. Elastin triblock copolymer hydrogel at 1,10, \& $25 \mathrm{wt} . \%$. Note $7 \mathrm{~nm}$ fibers (arrows). A Featureless * glass-like matrix constitutes the aqueous phase in the pore cavity.

FIG. 2. Self assembled amyloid fibers were suspended in a glass-like cryo-immobilized matrix *.

References

[1] J. Dubochet et al., Quarterly Review of Biophysics 21 (1988) 129.

[2] R.P. Apkarian et al., Microsc. Microanal. 9:4 (2003) 286.

[3] E.R. Wright et al., Microsc. Microanal. 9:3 (2003) 171.

[4] K. Nagapudi et al., Macromolecules 38 (2005) 345.

[5] The author gratefully acknowledges colleagues Drs. J. Dong, V.P. Conticello, E. Chaikof, \& E.R. Wright. 